\title{
Loose accumulation grain size distribution measurement based on digital image processing technology
}

\author{
Yong $\mathrm{He}$ \\ Key Laboratory of Ministry of Education for Hydraulic \\ and Water Transport Engineering \\ Chongqing Jiaotong University \\ Chongqing,China \\ e-mail:910517007@qq.com
}

\author{
Jinxiu Liu \\ School of River \& Ocean Engineering \\ Chongqing Jiaotong University \\ Chongqing,China \\ e-mail:1jxx0112@126.com
}

\begin{abstract}
Due to the large range of loose accumulation's grain size, traditional methods almost can not reasonably determine the grain size distribution.In this paper,there is a rapid measurement of its grain size distribution based on digital image processing technology. This method avoids the field sampling and laboratory tests. It cost lowly and fast, has an important significance for project construction.
\end{abstract}

Keywords-image processing; supervised classification; loose accumulation; grain size distribution

\section{INTRODUCTION}

With the deepening of China's rapid economic development and the western development strategy implemented, Hydro engineering such as ports, terminals, waterways geology as an important part of the national infrastructure construction extend to more complex mountains with seismology, bad climate and other conditions.Hydro engineering construction in the mountainous areas of complex, climate and other conditions often encountered this particular geological loose accumulation. The presence of loose accumulation will greatly affect Hydro project's construction costs and operating safety.This study shows that the soil physics, mechanics,water management, and other traits is closely related to the soil grain size distribution. The common method to determine the grain size distribution of the indoor test methods for fine-grained soil,sand,gravel,soil can guarantee the reliability of the experimental technique has been normative operating standards, experimental outcomes. The mountain projects(such as the construction of ports, terminals, waterways, highways, railways, etc.)often encountered loose accumulation, traditional test methods almost no way to achieve a reasonable determination of the grain size distribution.Mainly due to sampling difficulties and the soil particles grain size overrun,and the traditional test method has a high cost,long cycle,the results of low reliability deficiencies.For loose accumulation,rapid method for determining grain size distribution on digital image processing technology,can avoid the on-site sampling and laboratory tests, and low cost, fast, has an important significance for project construction.

\section{LOOSE ACCUMULATION GRAIN SIZE DISTRIBUTION MEASUREMENT IMPLEMENTATION PROCESS}

The loose accumulation grain size distribution measurement process including: field data acquisition, photo geometric correction to establish the relative threedimensional, edge detection, vector edge, build Topology, statistical area and calculate the equivalent grain size distribution.

\section{A. Field data acquisition}

In this article, the subject is a loose hillside after the earthquake in Wenchuan County.Inherent Nikon D300 SLR Digital Camera:13.1 million pixels, like pieces of $23.6 \times$ $15.8 \mathrm{~mm}(4288 \times 2848$ pixels, pixel size of $5.5 \mu \mathrm{m})$ and a focal length of $50 \mathrm{~mm}$ fixed focus lens to shoot.Accordance with the requirements of the test accuracy, the photographer obtain the test data from two directions, the same distance that the heap stones repeatedly shooting photographs. Then select clearer and representative images to facilitate the processing of data within the industry, and loose accumulation in Figure 1 below.

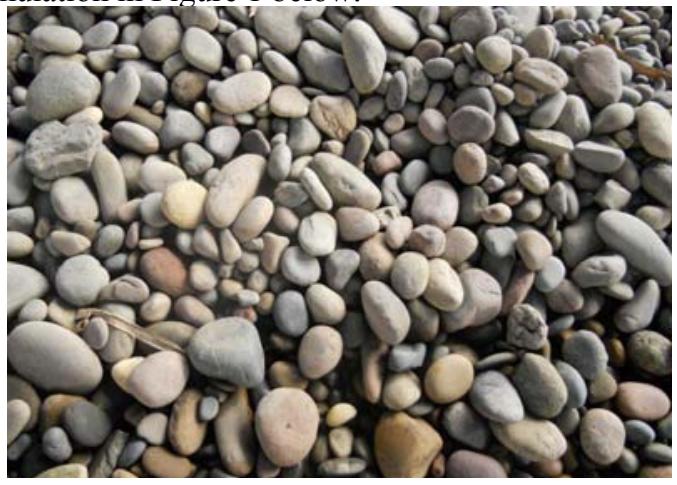

Figure 1. The example of loose accumulation in Wenchuan

\section{B. Establish three-dimensional image geometry correction relatively}

The actual shooting of the center point of the object with the camera will not produce geometric offset,the more away from the camera center point geometry offset the greater relative decrease. This decrease the error through the creation of three-dimensional.This process run at the Autosync 
module of Leica ERDAS.Adding two overlap adjacent image greater than $60 \%$ to the Autosync module.Selected five of the same name, the registration module automatically generates 26 matching points of the same name.Perspective relative alignment of the image shown in Figure 2:

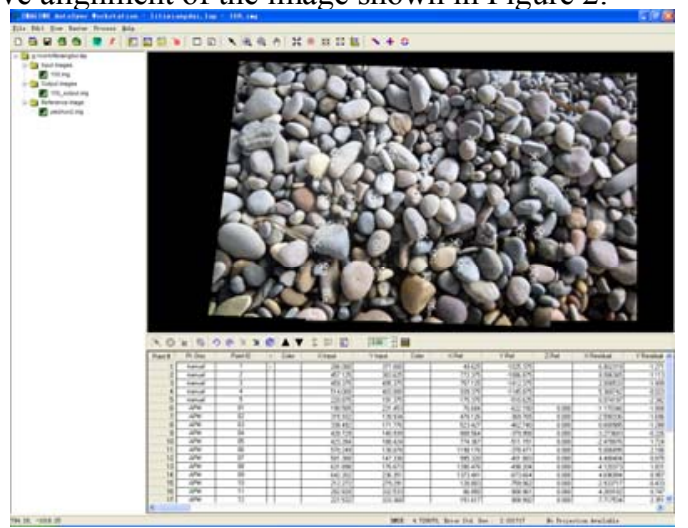

Figure 2. The result of registration

\section{Image preprocessing}

The edge detection algorithm is based on image intensity a first order derivative and a second order derivative number.But the derivative for noise is very sensitive.If not do smoothing processing for the enter image, it will detect too many pseudo edges in edge detection. The commonly filtering methods are neighborhood averaging method,the weighted average method, median filter,low-pass filtering,adaptive filtering method and so on.In this paper use the low-pass filtering of the $3 * 3$ Gaussian template to smooth image $3 * 3$ Gaussian is template as follows:

$$
\frac{1}{16}\left[\begin{array}{ccc}
1 & 2 & 1 \\
2 & 4 * & 2 \\
1 & 2 & 1
\end{array}\right]
$$

The image after filtering process shown in Figure 3:

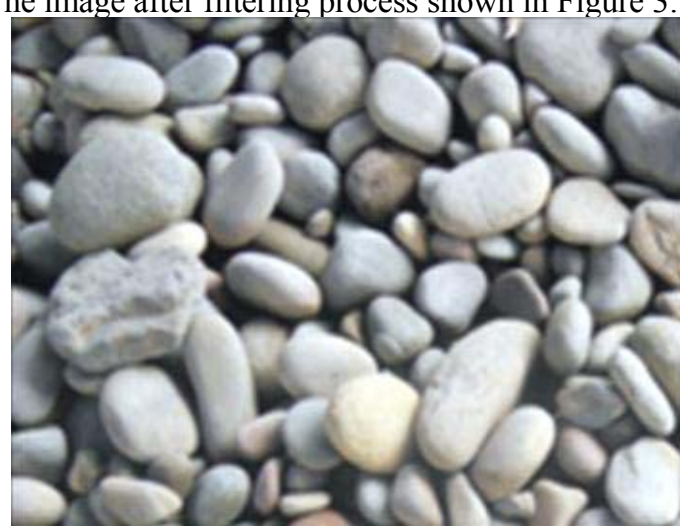

Figure 3. Gauss filter

Most filter can lead to loss of image edge information while smoothing noise.So it also need to do image enhancement after smoothing. Edge enhancement results shown in Figure 4:

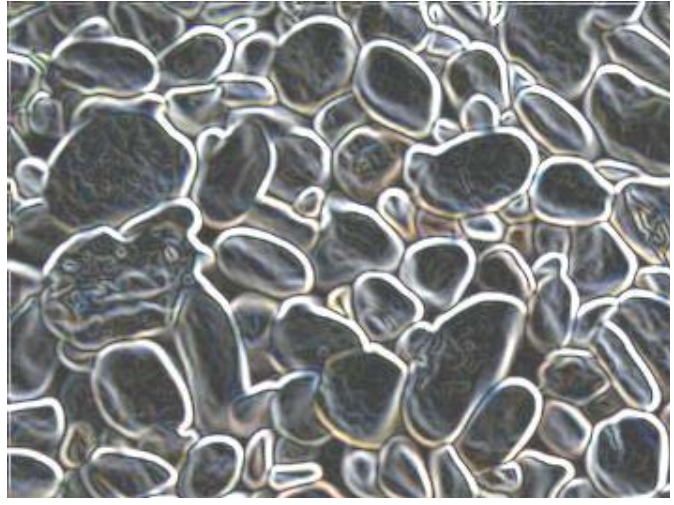

Figure 4. edge enhance

\section{Supervised classification edge extraction}

Supervised classification is also known as training venues method.It is based on the theoretical basis of the establishment of statistical identification function, the classification of technical training method based on a representative sample[1].That is based on samples of known training area, by selecting the characteristic parameters obtained characteristic parameters as the decision-making rules,discriminant function to each be classified images,image classification.It is a method of pattern recognition.

The common supervised classification algorithms are parallel hexahedron law, the maximum likelihood method[2,3],the minimum distance method[4],Mahalanobis distance[5],neural network classification method.The maximum likelihood method is the most typical application and one of the most widely supervised classification method.This article uses this method to do supervised classification.Classification results shown in Figure 5:

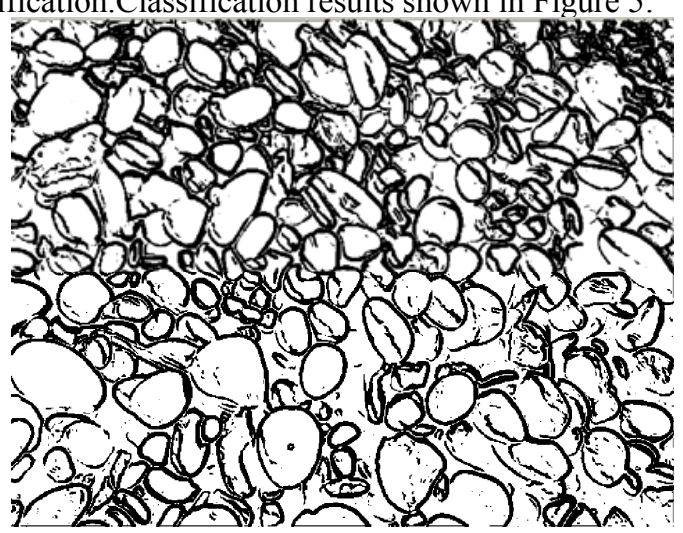

Figure 5. Maximum likelihood

\section{E. Build Topology}

Edge are effectively extracted by supervised classification, but it is raster images, and can not be edited,even in some software can make simple edits, but only the most basic operation. Build Topology can not be achieved.So it need to change raster images into vector 
layers. Vector layers and the original image superimposed results shown in Figure 6:

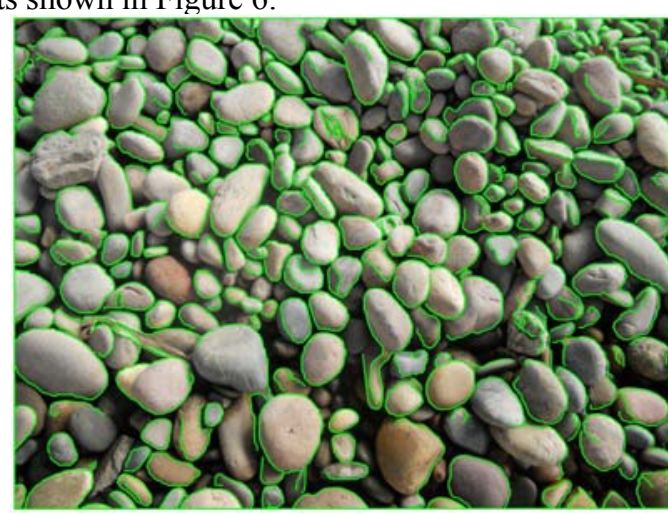

Figure 6. Vector layer add to original image

From the figure to see the edge of the vast majority of stones were extracted, and only a small portion is shaded place could not be extracted, which requires manual editing later, extract all stone edge, and then build Topology in the edit module of MAPGIS.

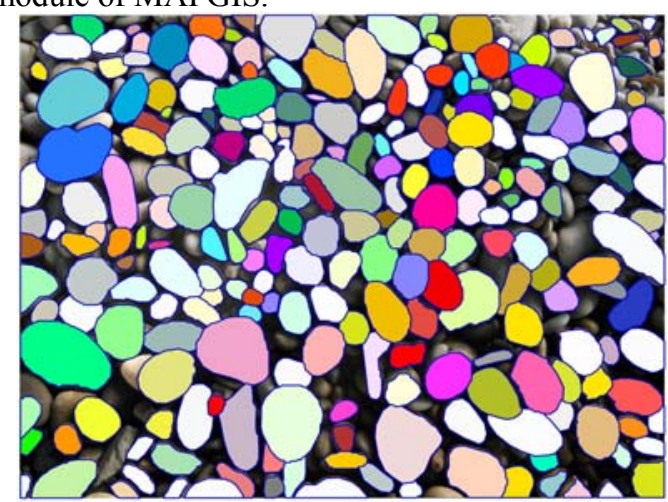

Figure 7. Topology layer add to original image

Before build Topology it is required to do the following assumptions:

- Many stones will be covered some part by other stones.In order to minimize the measurement error of this reason it required to assume that a stone submajority of the area in the lower portion (not visible) in the upper part (can be seen with the majority of the area) equal probability.

- Some stones elongate,some of the elongate vertical stones embedded stones crevices,some flat on stones,assuming that these two types of stones placed equal probability, these two causes the measurement error cancel each other.

- If a stone is mostly covered by other stones,put the stones as a background.It is not included in the grain size distribution statistics.Build Topology after edit, result in Figure 7.

\section{F. Statistical area and calculate the equivalent radius}

Statistics the area,the maximum,minimum and average values in MapGIS.Area will be converted into the equivalent grain size in EXCEL.We know photo stone diameter:focal length=gravel actual diameter:object distance,the trials, the focal length $=$ object distance $=50 \mathrm{~mm}$, the photos on the stones diameter=actual diameter of the stones, do not need to be converted.

TABLE I. EQUIVALENT GRAIN SIZE DISTRIBUTION

\begin{tabular}{|c|c|c|c|}
\hline Sub-area(unit:mm²) & $\begin{array}{c}\text { Equivalent } \\
\text { grain size }\end{array}$ & $\begin{array}{c}\text { Num } \\
\text { ber }\end{array}$ & $\begin{array}{c}\text { Percentage } \\
\text { of total }\end{array}$ \\
\hline $45.6422-2438.5008$ & $7.62-55.72$ & 188 & $76.4 \%$ \\
\hline $2438.5008-4831.3594$ & $55.72-78.43$ & 46 & $18.7 \%$ \\
\hline $4831.3594-7224.2180$ & $78.43-95.91$ & 8 & $3.3 \%$ \\
\hline $7224.2180-9617.0766$ & $95.91-110.66$ & 4 & $1.6 \%$ \\
\hline
\end{tabular}

In this picture there are 246 stones, the maximum area is $9617 \mathrm{~mm} 2$, minimum area is $46 \mathrm{~mm} 2$, average area is $1819 \mathrm{~mm} 2$.Corresponding equivalent diameter: maximum $111 \mathrm{~mm}$, minimum $8 \mathrm{~mm}$, average $48 \mathrm{~mm}$. The detailed equivalent diameter segmented as shown in Table 1.

Seen from the table:the vast majority of the total number of equivalent grain size between $7.62 \mathrm{~mm}-55.72 \mathrm{~mm}$ accounted for $76.4 \%$, followed by $55.72 \mathrm{~mm}-78.43 \mathrm{~mm}$ accounted for $18.7 \%$ of the total,greater than $78.43 \mathrm{~mm}$ only less than $5 \%$ of the total.The common pebbles in real life probably also about $10 \mathrm{~mm}-50 \mathrm{~mm}$ diameter,which is consistent to the test results and reality.Stones equivalent particle diameter of the specific distribution shown in Figure 8:

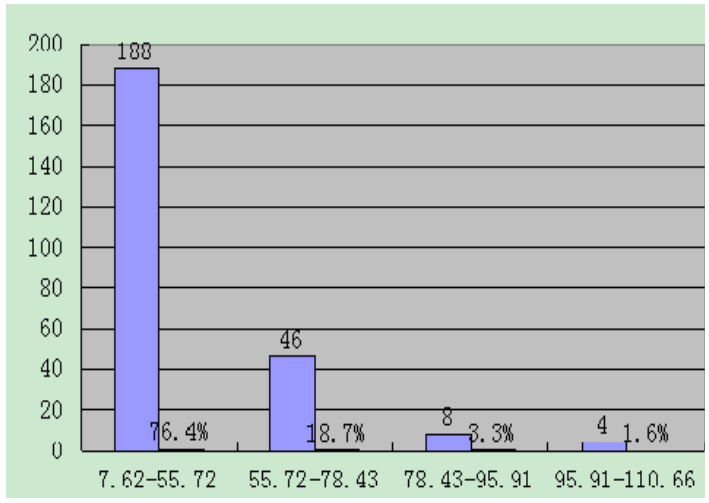

Figure 8. Equivalent diameter segmented maps

\section{CONCLUSION}

Equivalent grain size calculated based on digital image processing technology is in line with reality.The present method is more suitable for a grain size of the measured equivalent particle diameter of the larger $(>5 \mathrm{~mm})$,which exactly compensate for the deficiencies of the traditional laboratory sieving.This method avoids the field sampling and laboratory tests, and low cost,fast,has an important significance for project construction.

\section{ACKNOWLEDGMENT}

This work was supported by the Open Founds of Key Laboratory of Ministry of Education for Hydraulic and Water Transport Engineering (SLK2010B04), Chongqing Municipal Natural Science Foundation(CSTC, 
2011JJA30012) \& the Scientific and Technological Project of Chongqing Educational Committee(KJ110406).

\section{REFERENCES}

[1] Anxin Mei, "Introduction to Remote Sensing,"Beijing: Higher Education Press,2001.

[2] Yingshi Zhao, "Principles and methods of remote sensing application analysis," Beijing: Science Press,2003.

[3] John Rogan, "A comparison of method for monitoring multi emporia vegetation change using thematic mapper imagery," Remote sensing of environment.,2002.

[4] Zenglin Wang and Daming Zhu, "Algorithm discussion based on the maximum likelihood classification of remote sensing images," Henan Science, 2010,28 (11): :1458-1461.

[5] Pengxiao Chen and Shaohong Shen, "Terrestrial environmental change research based on multitemporal CBERS images," Yangtze river academy of sciences, 2011,28 (2) :70-73 